OPEN ACCESS

Edited by:

Xuguo Zhou,

University of Kentucky, United States

Reviewed by:

Hu Wan,

Huazhong Agricultural University,

China

Xiao-Qiang Yu,

South China Normal University, China

${ }^{*}$ Correspondence:

Keyan Zhu-Salzman

keyan.zhusalzman@ag.tamu.edu

Specialty section:

This article was submitted to Invertebrate Physiology,

a section of the journal

Frontiers in Physiology

Received: 11 December 2021 Accepted: 07 January 2022

Published: 28 January 2022

Citation:

Lei J, Tan Y, List F, Puckett $R$ Tarone AM, Vargo EL and Zhu-Salzman K (2022) Cloning and Functional Characterization of a Double-Stranded RNA-Degrading Nuclease in the Tawny Crazy Ant

(Nylanderia fulva).

Front. Physiol. 13:833652. doi: 10.3389/fphys.2022.833652

\section{Cloning and Functional Characterization of a Double-Stranded RNA-Degrading Nuclease in the Tawny Crazy Ant (Nylanderia fulva)}

\author{
Jiaxin Lei ${ }^{1}$, Yongan Tan ${ }^{1,2}$, Fabian List ${ }^{1}$, Robert Puckett ${ }^{1}$, Aaron M. Tarone ${ }^{1}$, \\ Edward L. Vargo ${ }^{1}$ and Keyan Zhu-Salzman ${ }^{1 *}$
}

${ }^{1}$ Department of Entomology, Texas A\&M University, College Station, TX, United States, ${ }^{2}$ Institute of Plant Protection, Jiangsu Academy of Agricultural Sciences, Nanjing, China

RNA interference is a powerful tool that post-transcriptionally silences target genes. However, silencing efficacy varies greatly among different insect species. Recently, we attempted to knock down some housekeeping genes in the tawny crazy ant (Nylanderia fulva), a relatively new invasive species in the southern United States, but only achieved relatively low silencing efficiency when dsRNA was orally administered. Here, we detected divalent cation-dependent, dsRNA-degrading activity in the midgut fluid of worker ants in ex vivo assays. To determine whether dsRNA degradation could contribute to low effectiveness of oral RNAi in N. fulva, we cloned its sole dsRNase gene (NfdsRNase). The deduced amino acid sequence contained a signal peptide and an endonuclease domain. Sequence alignment indicated a high degree of similarity with well-characterized dsRNases, particularly the six key residues at active sites. We also identified dsRNase homologs from five other ant species and found a tight phylogenetic relationship among ant dsRNases. NfdsRNase is expressed predominantly in the abdomen of worker ants. Oral delivery of dsRNA of NfdsRNase significantly reduced the expression of $N f d s R N$ ase transcripts, and substantially suppressed dsRNAdegrading activity of worker ants' midgut fluids as well. Our data suggest that dsRNA stability in the alimentary tract is an important factor for gene silencing efficiency in N. fulva, and that blocking NfdsRNase in gut lumen could potentially improve RNAi, a novel pest management tactic in control of $N$. fulva and other ant species.

Keywords: Nylanderia fulva, RNAi efficiency, dsRNA stability, dsRNase, midgut fluid

\section{INTRODUCTION}

The tawny crazy ant (Nylanderia fulva), native to South America, is an invasive species that has rapidly spread in the southern United States. It has caused significant economic and ecological damage since it was first reported in Texas in 2002. It is a nuisance to homeowners and businesses because of its damage to electrical systems in urban areas (Mcdonald, 2012). Nylanderia fulva 
also has the potential to cause significant agricultural damage by tending and protecting honeydew-producing insect pests in the field (Meyers, 2008; Mcdonald, 2012). Ecologically, due to its high fecundity and ability to form supercolonies (Eyer et al., 2018), this ant species is extremely competitive and threatens biodiversity by displacing native ant species and non-ant arthropods (Lebrun et al., 2013). Due to the extreme density of workers and the spatially expansive nature of their supercolonies, effective control of this ant has been difficult. Therefore, development of an efficient and environmental-friendly approach to control N. fulva populations is urgently needed.

Gene silencing mediated by double-stranded RNAs (dsRNAs) suppresses gene expression post-transcriptionally in a sequencespecific manner (Baum and Roberts, 2014; Cooper et al., 2019). RNA interference (RNAi) technology is becoming one of the most promising tools for gene function studies, as well as a means of pest management in the field (Kim et al., 2015; Allen, 2021). However, RNAi efficiency varies greatly among different insect species, strains, developmental stages or tissue types (Baum and Roberts, 2014; Cooper et al., 2019). While coleopterans are very susceptible to ingested dsRNA, lower RNAi efficiency has been observed in lepidopterans and hemipterans (Baum and Roberts, 2014). Our previous work showed rather modest effects of RNAi via dsRNA feeding in N. fulva (Meng et al., 2020). Of all house-keeping genes we selected, none exceeded 40\% reduction (Meng et al., 2020). Low potency is an obstacle in applying RNAi to management of $N$. fulva populations. Understanding the mechanisms underlying RNAi efficiency thus becomes imperative if RNAi is to be used as an effective control method.

Upon ingestion, dsRNA has to survive the hostile environment of the alimentary tract and enter cells in order to trigger the silencing machinery. Recent studies have demonstrated that degradation of dsRNA by dsRNAdegrading nucleases (dsRNase) may occur prior to cellular uptake. Extracellular degradation of dsRNA, notably in the gut, may explain the low efficiency of oral RNAi (Garbutt et al., 2013; Singh et al., 2017; Peng et al., 2018; Prentice et al., 2019; Cooper et al., 2020; Fan et al., 2020; Chen et al., 2021; Yoon et al., 2021). The first insect dsRNase identified in the midgut fluid of the domestic silk moth (Bombyx mori), contains a signal peptide and a non-specific endonuclease domain (Arimatsu et al., 2007). Ex vivo degradation assays showed that dsRNA stability can vary in the alimentary tracts of different insect species: dsRNA molecules persist longer in gut extracts of RNAi-sensitive red flour beetle (Tribolium castaneum) than in extracts from RNAi-insensitive pea aphid (Acyrthosiphon pisum) (Cao et al., 2018). Knockdown of dsRNase expression indeed greatly improves dsRNA stability in several insects across different orders, including the migratory locust (Locusta migratoria), the southern green stinkbug (Nezara viridula), the Queensland fruit fly (Bactrocera tryoni) and the Colorado potato beetle (Leptinotarsa decemlineata) (Song et al., 2017, 2019; Spit et al., 2017; Tayler et al., 2019; Sharma et al., 2021). Little research has been conducted to characterize dsRNases in ants.

We hypothesized that low RNAi efficacy in N. fulva was due to high dsRNase activity in the digestive tract, and that knockdown of its dsRNase enzymatic activity via gene silencing would significantly improve dsRNA stability. In this study, we attempted to detect dsRNA-degrading activity in worker ant midgut fluids. We cloned and characterized the sole dsRNase gene, $N f d s R N a s e$. In addition, we explored whether silencing $N f d s R$ Nase could prevent dsRNA from being degraded in the gut.

\section{MATERIALS AND METHODS}

\section{Insect Rearing}

Tawny crazy ant (N. fulva) colonies were collected from College Station, Texas, United States and maintained in an environmentally controlled room $\left(27^{\circ} \mathrm{C}, 80 \% \mathrm{RH}\right)$ following procedures reported previously with minor modifications (Meng et al., 2020). Colonies were reared in clear 32-L plastic storage bins with a lid, the vertical sides of which were coated with Fluon to prevent ants from escaping. Harborages inside the container were Petri dishes $(130 \mathrm{~mm} \times 25 \mathrm{~mm})$ with a layer $(\sim 5 \mathrm{~mm})$ of pre-wetted Castone ${ }^{\circledR}$ plaster (Dentsply, York, PA, United States) at the bottom. Artificial diet (3.5\% whey protein, 3.5\% whole egg powder, $3.5 \%$ cricket powder, $16.5 \%$ sucrose, $0.1 \%$ vitamin mix, $0.1 \%$ methylparaben and $2 \%$ agar), dead cockroaches, as well as clear culture tubes $(16 \mathrm{~mm} \times 150 \mathrm{~mm})$ half-filled with water or $13 \%$ sucrose, were provided to the colonies.

\section{Cloning the Full-Length NfdsRNase Gene}

BmdsRNase (NP_001091744.1), a well-characterized dsRNase from $B$. mori, was used as the query sequence in a TBLASTN search $^{1}$ against the $N$. fulva transcriptome database. To clone the full-length coding region of NfdsRNase, 50-60 worker ants were collected, frozen in liquid nitrogen and ground into fine power. Total RNA was extracted as described previously (Meng et al., 2020), and $2 \mu \mathrm{g}$ was used to synthesize cDNA by M-MuLV reverse transcriptase (NEB, Ipswich, MA, United States) according to the manufacturer's instructions with a gene-specific primer complementary to $3^{\prime}$ UTR of NfdsRNase (Table 1). The coding region was PCR-amplified using the above cDNA as templates and gene-specific primers (Table 1). The PCR product was purified by QIAquick ${ }^{\circledR}$ Gel Extraction Kit (QIAGEN) and its sequence was confirmed by sequencing analysis. The mRNA sequence was used to search against the $N$. fulva genome via BLASTN to determine the copy number of dsRNase.

\section{Analysis of Deduced Amino Acid Sequences}

NfdsRNase was used for transcriptome database searches to obtain mRNA sequences of other ant $d s R$ Nases from Camponotus floridanus, Solenopsis invicta, Atta cephalotes, Acromyrmex echinatior, and Linepithema humile by BLASTN. Deduced amino acid sequences ${ }^{2}$ were used to obtain molecular masses and isoelectric points (pI) of dsRNases using PROTEIN

\footnotetext{
${ }^{1}$ https://blast.ncbi.nlm.nih.gov/Blast.cgi

${ }^{2}$ http://web.expasy.org/translate/
} 
TABLE 1 | Primers used in this study.

\begin{tabular}{|c|c|c|}
\hline Purpose & Gene & Primer sequence $\left(5^{\prime}-3^{\prime}\right)$ \\
\hline cDNA cloning & NfdsRNase & $\begin{array}{l}\text { Forward: ggggGAGCTCTATAATGCTGGTGCCGAATGTGC } \\
\text { Reverse: ggggCTCGAGTTACATTAGAATGTCGATCACATCGAAC } \\
\text { 3' UTR: GACGGAATCAAGTGTTACTC }\end{array}$ \\
\hline \multirow[t]{2}{*}{ dsRNA synthesis } & NfdsRNase & $\begin{array}{l}\text { Forward: } \\
\text { TAATACGACTCACTATAGGGAGAGGACGACTTCATTACTTAGCA } \\
\text { Reverse: } \\
\text { TAATACGACTCACTATAGGGAGAGCGACATAATTCAATAGTGCGG }\end{array}$ \\
\hline & GFP & $\begin{array}{l}\text { Forward: } \\
\text { TAATACGACTCACTATAGGGAGAGTTCTGCTGGTAGTGGTCGG } \\
\text { Reverse: } \\
\text { TAATACGACTCACTATAGGGAGAGACGACGGCAACTACAAGAC }\end{array}$ \\
\hline RT-qPCR & NfdsRNase & $\begin{array}{l}\text { Forward: GCTCGCCACAATITCTCATCC } \\
\text { Reverse: TCGTTCTTGAGCCAACTCGG }\end{array}$ \\
\hline
\end{tabular}

CALCULATOR v3.4. ${ }^{3}$ SignalP-5.0 Server $^{4}$ and SMART domain analysis ${ }^{5}$ were used to predict the existence of signal peptides and conserved functional domains, respectively. WoLF PSORT computational web tool ${ }^{6}$ was used to predict subcellular localization. Amino acid sequence alignment of all six ant dsRNases were performed with Clustal X2.1 software (Chenna et al., 2003), and then visualized by GeneDoc software (Nicholas et al., 1997). The NCBI Conserved Domain Search ${ }^{7}$ identified active site, including $\mathrm{Mg}^{2+}$-and substrate-binding sites in the DNA/RNA non-specific endonuclease domains predicted.

Phylogenetic relationships of full-length amino acid sequences were analyzed using Maximum Likelihood method by MEGA 7.0 and tested by the bootstrap method with 1,000 replicates. NCBI accession numbers of protein sequences used to construct the phylogenetic tree are shown in Table 2.

\section{In vitro Synthesis of Double-Stranded RNAs}

Double-stranded RNAs were synthesized using in vitro transcription with T7 RNA polymerase (NEB) following the manufacturer's instructions with minor modification. The DNA templates for dsRNAs of green fluorescent protein (GFP) (dsGFP) and NfdsRNase (dsNfdsRNase) were PCR-amplified from pGL3-GFP and pET28a-NfdsRNase, respectively. T7 promoter sequences were added to $5^{\prime}$ ends of the primers (Table 1). PCR products were purified by QIAquick ${ }^{\circledR}$ Gel Extraction Kit (QIAGEN). Each reaction of in vitro transcription contained $1 \times$ RNAPol Reaction Buffer (NEB), $5 \mathrm{mM}$ DTT (NEB), $0.5 \mathrm{mM}$ rNTP (NEB), $1.25 \mathrm{U} / \mu \mathrm{L}$ T7 RNA polymerase (NEB), and $7.5 \mathrm{ng} / \mu \mathrm{L}$ DNA template. Reactions were incubated at $37^{\circ} \mathrm{C}$ overnight, and then treated by DNase I (NEB) and RNase One (Promega) simultaneously to remove DNA template and single-stranded RNA. dsRNA was purified by Ribozol ${ }^{\mathrm{TM}}$ reagent

\footnotetext{
${ }^{3} \mathrm{http}: / /$ protcalc.sourceforge.net/cgi-bin/protcalc

${ }^{4}$ http://www.cbs.dtu.dk/services/SignalP/

${ }^{5}$ http://smart.embl-heidelberg.de/

${ }^{6}$ https://www.genscript.com/wolf-psort.html

${ }^{7}$ https://www.ncbi.nlm.nih.gov/Structure/cdd/wrpsb.cgi
}

(VWR) following the manufacturer's instructions. dsRNA concentrations were determined by Nanodrop.

\section{Ex vivo Assays of Double-Stranded RNA Stability in Worker Ant Midgut Fluid}

Midguts were carefully dissected from worker ants under a dissecting microscope in pre-chilled $200 \mathrm{mM}$ sodium acetate at $\mathrm{pH} 4.5$, the physiological $\mathrm{pH}$. The midgut fluids from 10 guts were collected in $10 \mu \mathrm{L}$ of fresh dissection buffer. To do this, dissected guts were individually cut open with sharp forceps in the $10 \mu \mathrm{L}$ buffer, into which gut fluid was released. For each sample, gut fluids from at least 50 midguts were pooled from at least five such collections. Pooled samples were centrifuged at $16,000 \mathrm{~g}$ at $4^{\circ} \mathrm{C}$ for $15 \mathrm{~min}$. Supernatant was stored at $-20^{\circ} \mathrm{C}$ in $10 \mu \mathrm{L}$ aliquots until use. Protein concentration of the midgut fluids were determined by the Bradford assay (Bio-Rad, Hercules, CA, United States). Total proteins of $2 \mu \mathrm{g}$ were used for degrading assays.

To evaluate dsRNA stability, $200 \mathrm{ng}$ dsGFP was incubated with midgut fluids at $27^{\circ} \mathrm{C}$ for the time periods indicated, and then subjected to RNA extraction by RiboZol reagent (VWR) following the manufacturer's instructions. The isolated samples were dissolved in $8 \mu \mathrm{L}$ RNase-free water and examined on a 1.5\% agarose gel by ethidium bromide (EtBr) staining. Band intensity was quantified by Photoshop and expressed as a ratio relative to the sample that was prepared the same way but was not incubated at $27^{\circ} \mathrm{C}$.

\section{Feeding NfdsRNase Double-Stranded RNA to Worker Ants}

Worker ants were starved for $6 \mathrm{~h}$ prior to RNAi treatment. Forty of them were transferred to a plastic container $(20 \mathrm{~cm} \times 15 \mathrm{~cm} \times 10 \mathrm{~cm})$ with a fine brush. The vertical sides of the plastic container were coated with Fluon. Harborage was made with a culture tube $(13 \mathrm{~mm} \times 100 \mathrm{~mm})$ half-filled with water, which was plugged with a cotton ball and covered by aluminum foil. Water is also supplied by another culture tube without the aluminum foil cover. A wet cotton ball and three pieces of cardboard were placed in each container to retain 
TABLE 2 | NCBI accession numbers of amino acid sequences used to construct the phylogenetic tree.

\begin{tabular}{|c|c|c|c|}
\hline Order & Species & Protein name & Accession number \\
\hline \multirow[t]{6}{*}{ Diptera } & Aedes aegypti & AadsRNase & EAT42072.1 \\
\hline & Anopheles darlingi & AddsRNase1 & ETN62076.1 \\
\hline & & AddsRNase2 & ETN61460.1 \\
\hline & & AddsRNase3 & ETN61459.1 \\
\hline & Drosophila melanogaster & DmdsRNase1 & AAF49206.1 \\
\hline & & DmdsRNase2 & AAF49208.1 \\
\hline \multirow[t]{12}{*}{ Coleoptera } & Diabrotica virgifera virgifera & DvdsRNase1 & QNH88357.1 \\
\hline & & DvdsRNase2 & QNH88358.1 \\
\hline & & DvdsRNase3 & QNH88359.1 \\
\hline & Dendroctonus ponderosae & DpdsRNase1 & ENN82866.1 \\
\hline & & DpdsRNase2 & ERL84039.1 \\
\hline & & DpdsRNase3 & AEE63490.1 \\
\hline & Tribolium castaneum & TcdsRNase1 & QJD55726.1 \\
\hline & & TcdsRNase2 & QJD55727.1 \\
\hline & & TcdsRNase3 & QJD55728.1 \\
\hline & & TcdsRNase4 & QJD55729.1 \\
\hline & Leptinotarsa decemlineata & LddsRNase1 & APF31792.1 \\
\hline & & LddsRNase2 & APF31793.1 \\
\hline \multirow[t]{8}{*}{ Hymenoptera } & Nylanderia fulva & NfdsRNase & XP_029171190.1 \\
\hline & Camponotus floridanus & CfdsRNase & XP_011263277.1 \\
\hline & Solenopsis invicta & SidsRNase & XP_011156845.2 \\
\hline & Linepithema humile & LhdsRNase & XP_012230703.1 \\
\hline & Acromyrmex echinatior & AedsRNase & XP_011064189.1 \\
\hline & Atta cephalotes & AcdsRNase & XP_012064246.1 \\
\hline & Vespula vulgaris & VvdsRNase & KAF7408381.1 \\
\hline & Vespa mandarinia & VmdsRNase & XP_035717259.1 \\
\hline \multirow[t]{8}{*}{ Orthoptera } & Locusta migratoria & LmdsRNase1 & ARW74134.1 \\
\hline & & LmdsRNase2 & ARW74135.1 \\
\hline & & LmdsRNase3 & ARW74136.1 \\
\hline & & LmdsRNase4 & ARW74137.1 \\
\hline & Schistocerca gregaria & SgdsRNase1 & AHN55088.1 \\
\hline & & SgdsRNase2 & AHN55089.1 \\
\hline & & SgdsRNase3 & AHN55090.1 \\
\hline & & SgdsRNase4 & AHN55091.1 \\
\hline \multirow[t]{4}{*}{ Lepidoptera } & Bombyx mori & BmdsRNase1 & XP_012547127.1 \\
\hline & & BmdsRNase2 & NP_001091744.1 \\
\hline & Spodoptera littoralis & SldsRNase & CAR92522.1 \\
\hline & Spodoptera frugiperda & SfdsRNase & CAR92521.1 \\
\hline \multirow[t]{6}{*}{ Hemiptera } & Acyrthosiphon pisum & ApdsRNase & XP_003242652.1 \\
\hline & Diaphorina citri & DcdsRNase1 & XP_017297751.1 \\
\hline & & DcdsRNase2 & XP_008483858.1 \\
\hline & Cimex lectularius & CldsRNase1 & XP_014241898.1 \\
\hline & & CldsRNase2 & XP_014241376.1 \\
\hline & Frankliniella occidentalis & FodsRNase & XP_026279236.1 \\
\hline
\end{tabular}

moisture. For RNAi treatments, dsRNAs were administered orally via sucrose solution: treated $N$. fulva worker ants were fed on $13 \%$ sucrose solution containing $150 \mathrm{ng} / \mu \mathrm{L}$ dsNfdsRNase (30 $\mu \mathrm{L}$ in a PCR tube was put in each plastic container). The negative control consisted of ants fed on sucrose solution containing $150 \mathrm{ng} / \mu \mathrm{L}$ dsGFP. The dsRNA-containing sucrose solution, the only food source during the RNAi treatment, was replenished every 2 days. The ants were kept in the rearing room $\left(27^{\circ} \mathrm{C}, 80 \% \mathrm{RH}\right)$. After feeding on dsRNA-containing diet for 5 days, midgut fluids were collected as mentioned above for ex vivo assays of dsRNA stability and whole insects were harvested for gene expression analysis.

\section{Gene Expression Analysis of $\mathbf{N f d s R N a s e}$}

The expression of $N f d s R$ Nase was determined by quantitative RTPCR (RT-qPCR). Heads, thoraxes, and abdomens were dissected from 50 to $80 \mathrm{~N}$. fulva workers and instantly frozen in liquid nitrogen. For expression analysis of worker ants subjected to 
RNAi assays, entire bodies or abdomens of $\sim 40$ workers were pooled in each replicate of each treatment and frozen in liquid nitrogen. All samples for gene expression analysis were stored at $-80^{\circ} \mathrm{C}$ until further processing. Total RNA extraction and cDNA synthesis using random hexamer primers (Invitrogen, Carlsbad, CA, United States) were conducted as described above. Primers used in qPCR analysis were shown in Table 1 . The 60 S ribosomal protein L4 gene (NfRPL4) served as the internal control (Meng et al., 2020). qPCR reactions were run on a CFX384 Real-Time System (BioRad) using SYBR Green Mastermix reagent (BioRad). Relative fold changes of gene expression were calculated as we previously described (Zhu-Salzman et al., 2003).

\section{Statistical Analysis}

All statistical analyses in this study were performed by SPSS software (v.20.0; SPSS Inc., Chicago, IL, United States). Relative gene expression data were analyzed by the independent samples' Student's $t$ test.

\section{RESULTS}

\section{Midgut Fluids Exhibited Potent Double-Stranded RNA-Degrading Activities}

To determine if the midgut lumen of worker ants possessed dsRNA-degrading activity, we assessed the stability of dsGFP in midgut fluid, followed by RNA extraction and agarose gel electrophoresis. The ex vivo assays showed that dsGFP was gradually degraded when incubated with midgut fluids, and only $23 \%$ remained after $2 \mathrm{~h}$ of incubation (Figures 1A,B). The degradation speed was highest during the first $0.5 \mathrm{~h}$ of incubation and slowly decreased afterward (Figure 1B). Moreover, EDTA, the chelating agent, effectively blocked degradation of dsGFP in gut fluids (Figure 1C), suggesting that the dsRNA degrading activity of the ribonuclease is metal ion-dependent. As expected, the denatured nuclease lost its enzymatic activity (Figure 1C). These data support that midgut fluids exhibit dsRNA-degrading activity, which in turn could contribute to relatively low RNAi efficacy in ants observed in our previous manuscript (Meng et al., 2020).

\section{Identification and Characterization of a Nylanderia fulva Double-Stranded RNA-Degrading Nucleases Gene}

The presence of dsRNA-degrading activity in midgut fluid suggested the involvement of dsRNase. We searched the $N$. fulva transcriptome/genome and found only one dsRNase gene (NfdsRNase, XM_029315357). Multiple sequence alignment and functional domain analyses of NfdsRNase with wellcharacterized dsRNases from the domestic silk moth (B. mori), the African sweet potato weevil (Cylas puncticollis), the Colorado potato beetle ( $L$. decemlineata), the red flour beetle (T. castaneum), the silver leaf whitefly (Bemisia tabaci), and the migratory locust (L. migratoria) (Liu et al., 2012; Luo et al., 2017; Song et al., 2017; Spit et al., 2017; Prentice et al., 2019; Peng et al., 2020) revealed that NfdsRNase contained key properties
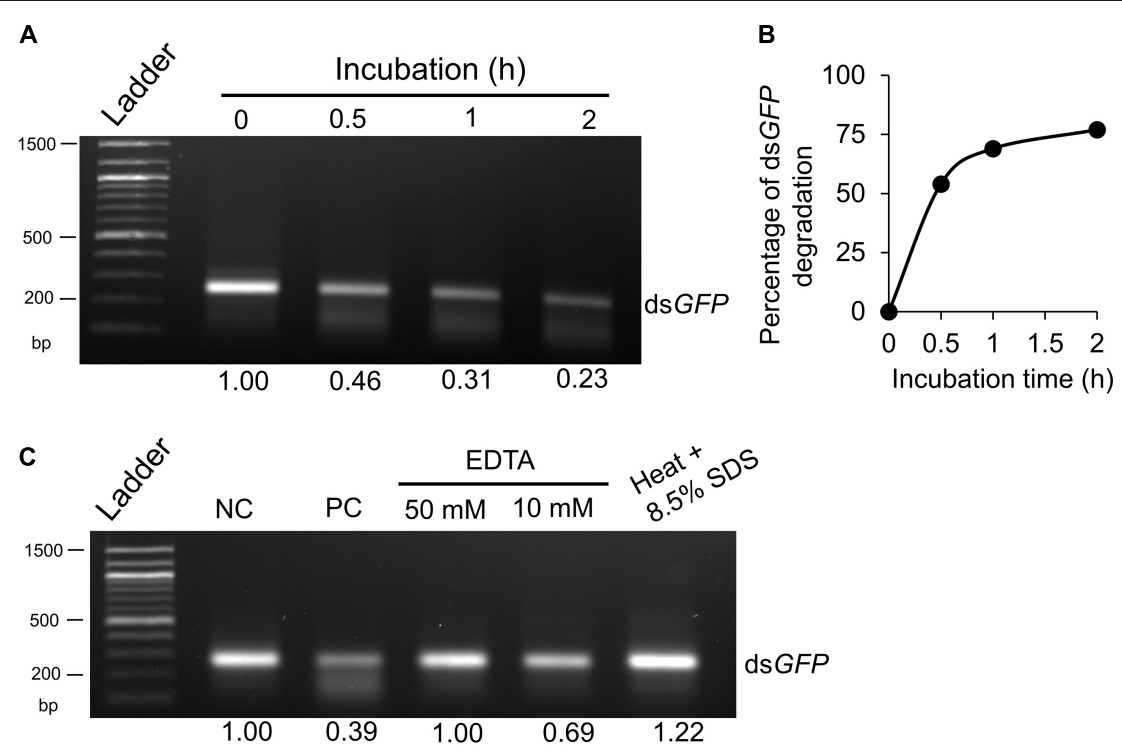

FIGURE 1 | Degradation of double-stranded RNA (dsRNA) ex vivo by midgut fluids of worker ants. (A) dsGFP (200 ng) was incubated with $2 \mu \mathrm{g}$ of midgut fluid proteins at $27^{\circ} \mathrm{C}$ for $0,0.5,1$ and $2 \mathrm{~h}$, respectively. (B) The percentage of dsGFP degradation in midgut fluids was calculated based on band intensity in panel (A). (C) dsRNA degradation in midgut fluids was inhibited by EDTA or heat + SDS treatment. dsGFP (200 ng) was incubated for 0 (negative control, NC) or $2 \mathrm{~h}$ (positive control, PC) at $27^{\circ} \mathrm{C}$ with midgut fluids as above. dsGFP and midgut fluids were also incubated in the presence of EDTA (50 and $10 \mathrm{mM}$ ), or heated at $95^{\circ} \mathrm{C}$ for 30 min in $8.5 \%$ SDS. After incubation, samples were subjected to RNA extraction by Ribozol, separated on 1.5\% agarose gel and visualized by EtBr staining under UV light. Band intensity was quantified by Photoshop and expressed as a ratio relative to the "0" or "negative control," which was arbitrarily set at 1. Each experiment was repeated at least twice and a representative gel picture was presented. 
A

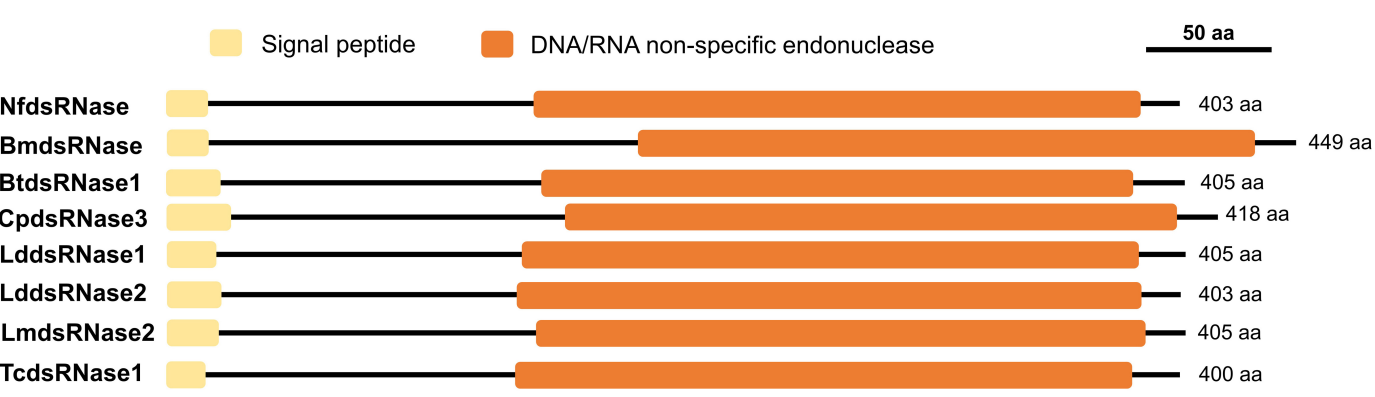

B
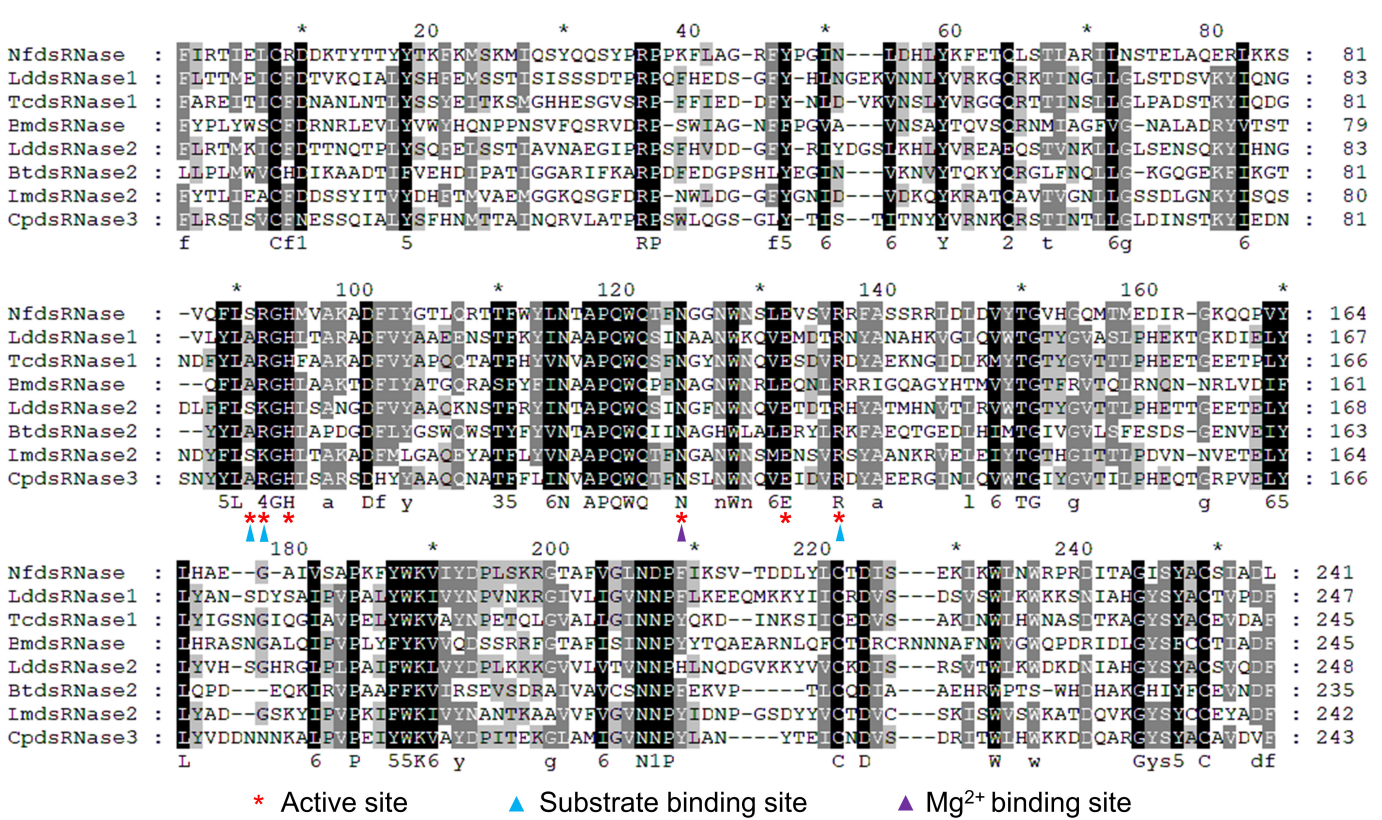

FIGURE 2 | Analysis of deduced amino acid sequences of NfdsRNases with well-characterized dsRNases from other insects. (A) Schematic diagrams of key function domains of dsRNases. Yellow boxes indicate predicted signal peptides; Orange boxes denote the DNA/RNA non-specific endonuclease domains. (B) Multiple sequence alignment of the DNA/RNA non-specific endonuclease domains in insect dsRNases (Nf, Nylanderia fulva; Bm, Bombyx mori; Bt, Bemisia tabaci; Cp, Cylas puncticollis; Lm, Locusta migratoria; Ld, Leptinotarsa decemlineata; Tc, Tribolium castaneum). Red asterisks indicate the active sites. Substrateand $\mathrm{Mg}^{2+}$-binding-sites are marked with blue and purple triangles, respectively. Numbers under the aligned sequences denote amino acid similarity groups of DN, EQ, ST, KR, FYW, and LIVM, respectively.

TABLE 3 | Characterization of NfdsRNase and seven previously reported insect dsRNases.

\begin{tabular}{|c|c|c|c|c|c|c|c|c|}
\hline Name & Species & $\begin{array}{c}\text { NCBI accession } \\
\text { number }\end{array}$ & $\begin{array}{c}\text { Number of amino } \\
\text { acids (aa) }\end{array}$ & $\begin{array}{c}\text { Molecular } \\
\text { weight }^{1} \text { (kDa) }\end{array}$ & $\begin{array}{l}\text { Isoelectric } \\
\text { point }^{1}(\mathrm{pl})\end{array}$ & $\begin{array}{l}\text { Signal peptide } \\
\text { cleavage site } \\
\text { (aa) }\end{array}$ & $\begin{array}{l}\text { DNA/RNA non-specific } \\
\text { endonuclease domain }{ }^{3} \text { (aa) } \\
\text { and similarity }(\%)^{4}\end{array}$ & $\begin{array}{l}\text { Subcellular } \\
\text { localization }^{5}\end{array}$ \\
\hline NfdsRNase & Nylanderia fulva & XP_029171190 & 403 & 46.0 & 9.03 & $16-17$ & 146-386 (100\%) & Extracellular \\
\hline BmdsRNase & Bombyx mori & NP_001091744.1 & 449 & 50.9 & 9.35 & $16-17$ & 188-432 (34.0\%) & Extracellular \\
\hline BtdsRNase2 & Bemisia tabaci & XP_018913086.1 & 405 & 45.1 & 8.05 & $21-22$ & 150-384 (31.9\%) & Extracellular \\
\hline CpdsRNase3 & Cylas puncticollis & QCF41177.1 & 418 & 47.2 & 6.18 & $25-26$ & $159-401$ (32.9\%) & Extracellular \\
\hline LddsRNase1 & $\begin{array}{l}\text { Leptinotarsa } \\
\text { decemlineata }\end{array}$ & APF31792.1 & 405 & 45.0 & 8.37 & $19-20$ & $141-387$ (34.0\%) & Extracellular \\
\hline LddsRNase2 & & APF31793.1 & 403 & 45.6 & 6.98 & $21-22$ & 139-386 (36.3\%) & Extracellular \\
\hline LmdsRNase2 & $\begin{array}{l}\text { Locusta } \\
\text { migratoria }\end{array}$ & ARW74135.1 & 405 & 43.8 & 4.98 & $21-22$ & $147-388$ (42.4\%) & Extracellular \\
\hline TcdsRNase1 & $\begin{array}{l}\text { Tribolium } \\
\text { castaneum }\end{array}$ & QJD55726.1 & 400 & 44.4 & 4.97 & $15-16$ & 139-383 (36.5\%) & Extracellular \\
\hline
\end{tabular}

${ }^{1}$ Molecular weight and isoelectric point was predicted based on the PROTEIN CALCULATOR v3.4 (http://protcalc.sourceforge.net/cgi-bin/protcalc).

${ }^{2}$ Signal peptide cleavage site was predicted according to the SignalP-5.0 Server (http://www.cbs.dtu.dk/services/SignalP/).

${ }^{3}$ Endonuclease_NS domain was predicted based on NCBI Conserved Domains (https://www.ncbi.nlm.nih.gov/Structure/cdd/wrpsb.cgi).

${ }^{4}$ Percentage of similarity to amino acid sequence of NfdsRNase in the DNA/RNA non-specific endonuclease domain was calculated by NCBI BLASTP suite-2sequences.

${ }^{5}$ Subcellular localization was predicted by the WoLF PSORT Prediction (https://www.genscript.com/wolf-psort.html).

bp, base pair; kDa, kilodalton; aa, amino acid. 


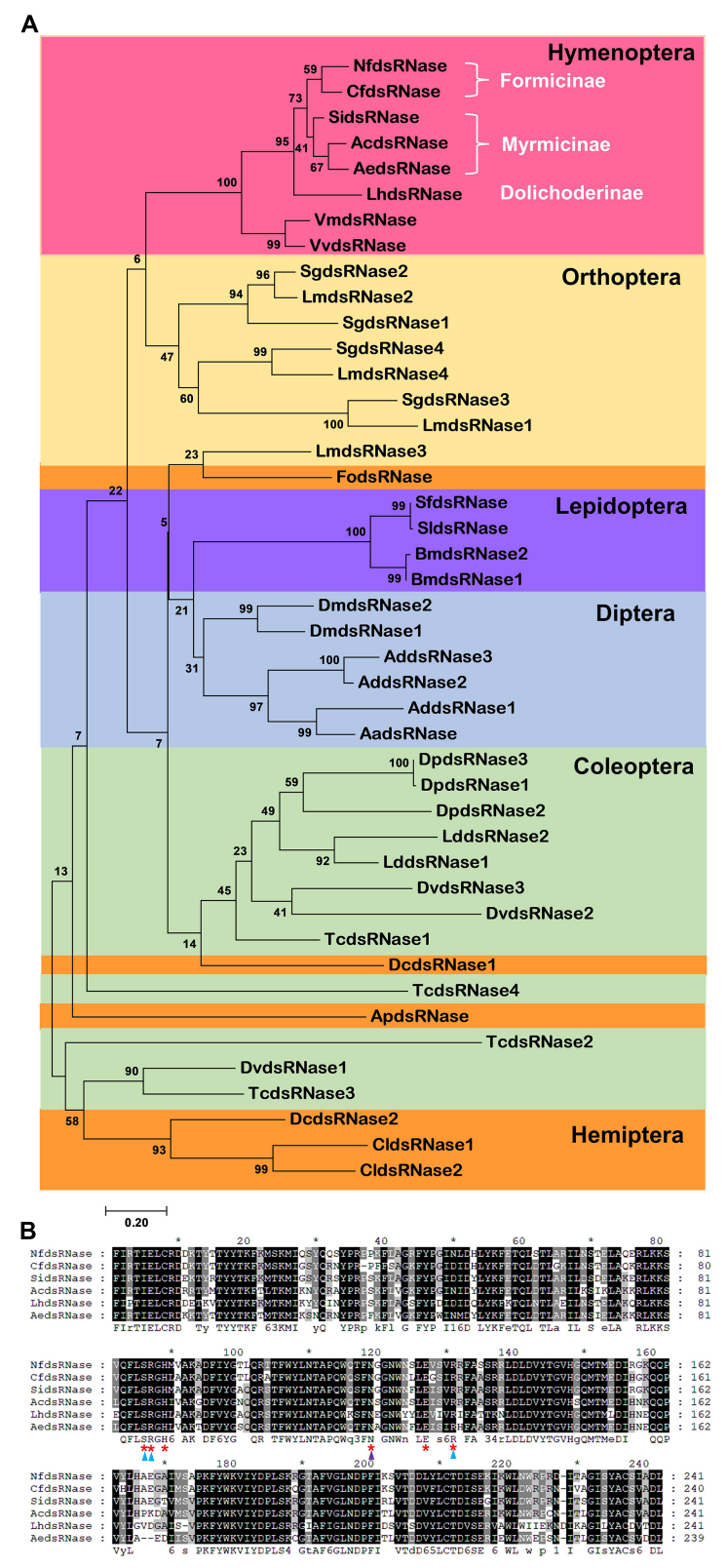

FIGURE 3 | Phylogenetic analysis of insect dsRNases. (A) The phylogenetic tree of dsRNase proteins from insects belonging to six orders was constructed by MEGA 7.0 using Maximum Likelihood method. The confidence values (\%) of each branch on the phylogenetic trees were calculated based on 1000 bootstrap replicates. Different shadings represent different insect orders. Coleoptera: Dp, Dendroctonus ponderosae; Dv, Diabrotica virgifera virgifera; Ld, Leptinotarsa decemlineata; Tc, Tribolium castaneum. Diptera: Aa, Aedes aegypti; Ad, Anopheles darling; Dm, Drosophila melanogaster. Hemiptera: Ap, Acyrthosiphon pisum; Cl, Cimex lectularius; Dc, Diaphorina citri; Fo, Frankliniella occidentalis. Hymenoptera: Ac, Atta cephalotes; Ae, Acromyrmex echinatior; Cf, Camponotus floridanus; Lh, Linepithema humile; Nf, Nylanderia fulva; Si, Solenopsis invicta.; Vv, Vespula vulgaris; Vm, Vespa mandarinia. Subfamilies are also marked. Lepidoptera: Bm, Bombyx mori; Sf, Spodoptera frugiperda; SI, Spodoptera littoralis. Orthoptera: Lm, Locusta migratoria; Sg, Schistocerca gregaria. (B) Multiple sequence alignment of the DNA/RNA non-specific endonuclease domains in ant dsRNases. Red asterisks indicate the active sites. Substrate- and $\mathrm{Mg}^{2+}$-binding-sites are marked with blue and purple triangles, respectively.

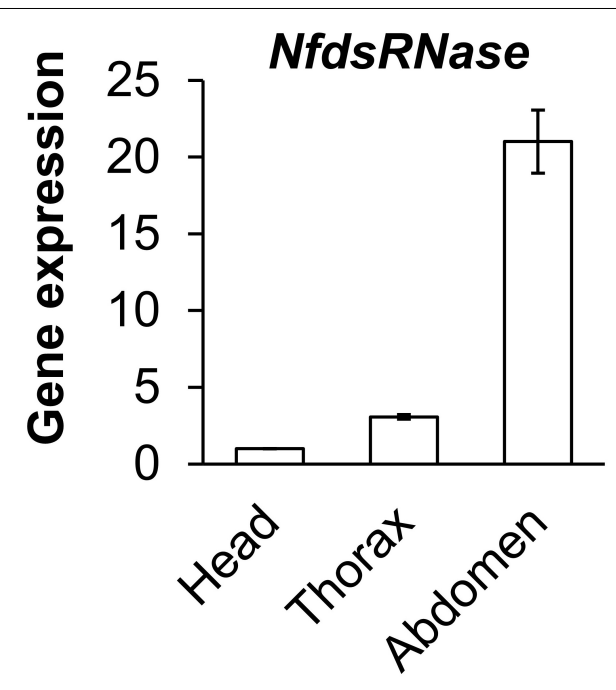

FIGURE 4 | Relative expression of NfdsRNase in head, thorax and abdomen of worker ants. Gene expression was determined by RT-qPCR, with $60 S$ ribosomal protein $L 4$ served as the internal control.

of a classic dsRNase. Similar to previously reported dsRNases, NfdsRNase contained a signal peptide (16 amino acids in length) and a highly conserved DNA/RNA non-specific endonuclease domain (Table 3 and Figures 2A,B). In the endonuclease domain of NfdsRNase, the six residuals at its putative active site were highly conserved, including substrate- and $\mathrm{Mg}^{2+}$-binding sites (Figure 2B). These molecular characteristics supported the extracellular nuclease nature of NfdsRNase.

Given the wide distribution of dsRNases in insects (Peng et al., 2020), it would be interesting to know their phylogenetic relationships. We performed phylogenetic analysis on 44 dsRNase protein sequences from 24 insect species from Coleoptera, Diptera, Hemiptera, Hymenoptera, Lepidoptera, and Orthoptera (Figure 3A). NfdsRNase was in the same clade with homologs from Hymenoptera, and consistent with their taxonomic relationship within ants (Ward, 2014). Notably, the six residues at the active site of the endonuclease domain were identical in all ants (Figure 3B). Homologs from Orthoptera were most closely related to those from Hymenoptera. Notably, dsRNases from Hemiptera appeared to be diverse.

\section{High Expression of $\mathbf{N f d s R N a s e}$ in the Abdomen of Worker Ants}

We analyzed NfdsRNase expression in different segments of worker ants (head, thorax, and abdomen) by RT-qPCR (Figure 4). The highest expression was seen in abdomen, followed by thorax and head. The NfdsRNase transcript in abdomen was $>20$-fold higher than that in the head, consistent with previous reports in other insect species that dsRNase is predominant expressed in insect guts (Peng et al., 2020; Chen et al., 2021). Enriched NfdsRNase transcripts in abdomen suggested that $N f d s R N a s e$ was most likely present in the digestive tract of N. fulva. 
A

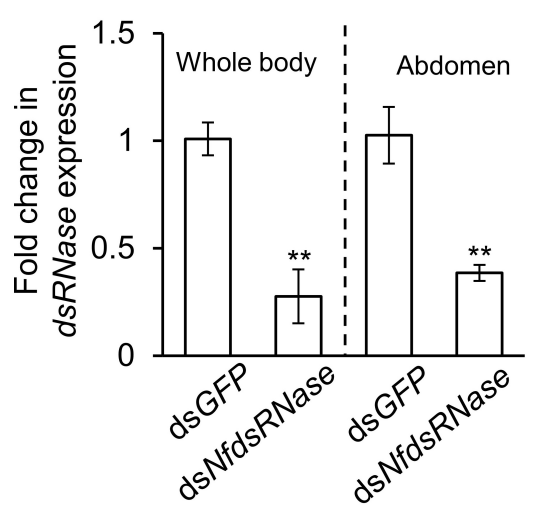

B

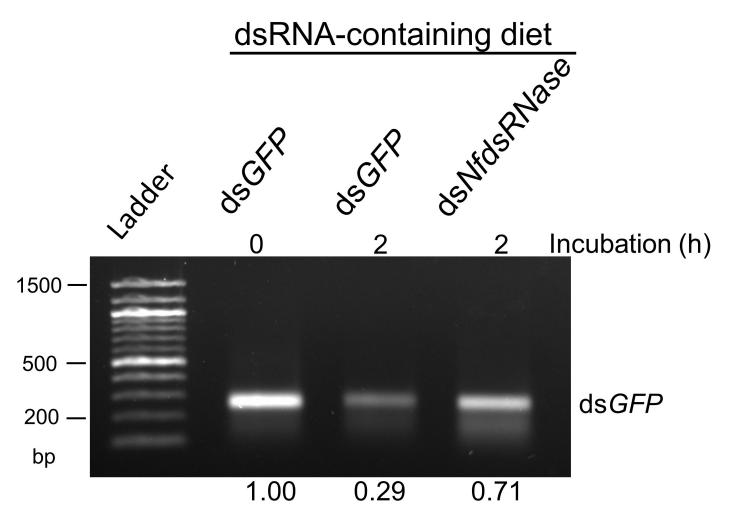

dsRNA-containing diet

FIGURE 5 | Contribution of NfdsRNase to dsRNA-degrading activity in worker ant midgut. After feeding worker ants with $13 \%$ sucrose solution containing $150 \mathrm{ng} / \mu \mathrm{L}$ dsGFP or dsNfdsRNase for 5 days, samples (whole body and abdomen) were harvested for gene expression analysis and midgut fluids were collected for ex vivo assays. Feeding on dsNfdsRNase-containing diet significantly reduced the expression of NfdsRNase (A) and dsRNase activity in midgut fluids (B) in worker ants. Gene expression analysis and ex vivo assays were performed as described above. Relative gene expression data were analyzed by the independent samples' Student's $t$ test. Statistical significance is marked by ${ }^{\star \star} P<0.01$. dsRNAs incorporated into the $13 \%$ sucrose diet were shown above the gel image and duration of ex vivo incubation with dsGFP was also indicated.

\section{Knockdown of NfdsRNase Expression Significantly Reduced Double-Stranded RNA-Degrading Activity in Midgut Fluids}

To determine whether $N f d s R$ Nase was responsible for dsRNA degradation, we fed worker ants the dsRNA specific to $N f d s R$ Nase (dsNfdsRNase) incorporated into 13\% sucrose solution. Workers fed on dsGFP-containing sucrose solution served as the negative control. Ingestion of dsNfdsRNase led to 72 and $61 \%$ transcript reduction in whole body and abdomen, respectively (Figure 5A). Incubation of dsGFP with midgut fluids collected from workers fed on diets containing dsNfdsRNase resulted in a notable $42 \%$ increase of dsRNA stability when compared to insects fed on dsGFP control (Figure 5B). Our results strongly supported that NfdsRNase was a key enzyme involved in dsRNA degradation in the midgut lumen.

\section{DISCUSSION}

RNAi-based control measures have great potential to be developed into environmental-friendly, species-specific insecticides (Whyard et al., 2009). Oral delivery of dsRNA is the most suitable for field applications due to feasibility and scalability (Peng et al., 2020; Allen, 2021). While this method has shown promise in some cases (Baum et al., 2007; Mao et al., 2011), it is less effective in triggering RNAi responses in other insects, such as N. fulva (Meng et al., 2020). Ingested dsRNAs have to undergo a number of physiological barriers prior to exerting their gene silencing action. Degradation by dsRNase in the digestive system is considered the first hurdle that dsRNAs come across (Cooper et al., 2019). In this study, we cloned and characterized the sole $N f d s R N a s e$ gene from $N$. fulva. We not only showed its highest expression in abdomen, but also revealed the dsRNA-degrading activities of its protein product in midgut fluids of the worker ants. This is the first biochemical and genetic study of dsRNases in an ant species.

We initially proposed that degradation of dsRNA by NfdsRNase in the midgut lumen could be one of the limiting factors for RNAi efficiency through oral dsRNA delivery in $N$. fulva, and our data supported this hypothesis. Sequencing alignment revealed that NfdsRNase belonged to a superfamily of nucleases that degrade dsRNA (Friedhoff et al., 1994; Figures 2, 3). Key residues at the catalytic sites of the functional domain in NfdsRNase shared high similarity with previously characterized dsRNases (Liu et al., 2012; Song et al., 2017; Peng et al., 2020; Figure 2). In addition, the presence of the signal peptide (Figure 2 and Table 3 ) and high expression in the abdomen (Figure 3) indicated that NfdsRNase was likely an extracellular nuclease secreted into the midgut lumen. Moreover, inhibition of dsRNA-degrading activity by EDTA suggested that divalent cations play an important role in NfdsRNasemediated nucleotide cleavage, consistent with other dsRNases (Cooper et al., 2020; Peng et al., 2020). Since silencing NfdsRNase resulted in a robust reduction of dsRNase activity (Figures 1, 5), degradation of dsRNA in midgut fluids was due largely to NfdsRNase in ants, which is also consistent with findings in other insects. Expression of functional recombinant NfdsRNase protein will allow us to learn additional properties, including substrate specificity, e.g., ssRNA, dsRNA, ssDNA, dsDNA, and circular plasmids.

Interestingly, there is only one copy of the dsRNase gene in all ant species we analyzed in this study, whereas most insect species possess at least two dsRNase genes. For example, L. decemlineata has two, L. migratoria has four, and the yellow fever mosquito (Aedes aegypti) have ten (Song et al., 2017; Spit et al., 2017; Giesbrecht et al., 2020). Multiple dsRNase genes are believed to be related to tissue-specific expression, functional divergence, and adaptation 
to various environments (Song et al., 2017, 2019; Giesbrecht et al., 2020). For instance, in L. migratoria, LmdsRNase1 and LmdsRNase4 are mainly expressed in hemolymph; while LmdsRNase 2 and LmdsRNase 3 are predominant in midgut. Among them, only LmdsRNase2 is a major contributor to dsRNA degradation (Song et al., 2017, 2019). It would be interesting to compare the enzymatic activity, substrate specificity, and environmental response between NfdsRNase and dsRNases from insects with multiple copies. In addition, ant dsRNases display remarkable sequence conservation with identical active site residues (Figure 3). Since variations in the first and second substrate binding sites have been long suggested to be related to differential substrate specificity (Wynant et al., 2014; Song et al., 2017, 2019), identical active site residues may imply that they share similar ribonuclease activities and thus the knowledge obtained from this research could be applicable to other ant species.

Recent studies have provided solid evidence that silencing dsRNase genes could significantly improve RNAi efficiency in a number of insects, including the African sweet potato weevil, the Colorado potato beetle, the red flour beetle, the silver leaf whitefly, the southern green stink bug, the migratory locust and the Queensland fruit fly (Luo et al., 2017; Song et al., 2017; Spit et al., 2017; Prentice et al., 2019; Tayler et al., 2019; Peng et al., 2020; Sharma et al., 2021). Therefore, knockdown of NfdsRNase should also lead to enhanced N. fulva RNAi efficacy. Additionally, alternative delivery systems-mediated by nanoparticles, such as chitosan, liposomes, and cationic dendrimers (Zhang et al., 2010; Zheng et al., 2019; Wang et al., 2020), should also be explored in future studies to assess their effectiveness to prevent dsRNA degradation and improve RNAi efficiency.

\section{REFERENCES}

Allen, M. L. (2021). Prospects for using RNAi as control for ants. Front. Agron. 2021:591539.

Arimatsu, Y., Kotani, E., Sugimura, Y., and Furusawa, T. (2007). Molecular characterization of a cDNA encoding extracellular dsRNase and its expression in the silkworm, Bombyx mori. Insect Biochem. Mole. Biol. 37, 176-183. doi: 10.1016/j.ibmb.2006.11.004

Baum, J. A., Bogaert, T., Clinton, W., Heck, G. R., Feldmann, P., Ilagan, O., et al. (2007). Control of coleopteran insect pests through RNA interference. Nat. Biotechnol. 25, 1322-1326. doi: 10.1038/nbt1359

Baum, J. A., and Roberts, J. K. (2014). Progress towards RNAi-mediated insect pest management. Insect Midgut Insect. Prot. 47, 249-295.

Cao, M., Gatehouse, J. A., and Fitches, E. C. (2018). A systematic study of RNAi effects and dsRNA stability in Tribolium castaneum and Acyrthosiphon pisum, following injection and ingestion of analogous dsRNAs. Int. J. Mol. Sci. 2018:19. doi: $10.3390 /$ ijms19041079

Chen, J. Z., Jiang, Y. X., Li, M. W., Li, J. W., Zha, B. H., and Yang, G. (2021). Double-stranded RNA-degrading enzymes reduce the efficiency of RNA interference in Plutella xylostella. Insects 2021:12. doi: 10.3390/insects120 80712

Chenna, R., Sugawara, H., Koike, T., Lopez, R., Gibson, T. J., Higgins, D. G., et al. (2003). Multiple sequence alignment with the Clustal series of programs. Nucleic Acids Res. 31, 3497-3500. doi: 10.1093/nar/gkg500

Cooper, A. M., Silver, K., Zhang, J., Park, Y., and Zhu, K. Y. (2019). Molecular mechanisms influencing efficiency of RNA interference in insects. Pest Manag. Sci. $75,18-28$. doi: 10.1002/ps.5126
In summary, our NfdsRNase study is consistent with previously findings of several well-characterized dsRNase from other insects. Rapid degradation of dsRNA in midgut fluids is likely the key factor for modest RNAi effectiveness observed in our previous study (Meng et al., 2020). While it is important to enhance dsRNA stability in the digestive tract, other potential issues, such as limited cellular uptake, weak systemic transport, and insufficient RNAi core machinery need to be addressed (Cooper et al., 2019; Zhu and Palli, 2020) to develop effective RNAi strategies for control of $N$. fulva and other ant species.

\section{DATA AVAILABILITY STATEMENT}

The original contributions presented in the study are included in the article/supplementary material, further inquiries can be directed to the corresponding author.

\section{AUTHOR CONTRIBUTIONS}

JL, RP, AT, EV, and KZ-S designed the experiments. JL, YT, and FL performed the experiments. JL and KZ-S wrote the manuscript. All authors contributed to the article and approved the submitted version.

\section{FUNDING}

This study received financial support from Invasive Ant Research and Management Project.

Cooper, A. M. W., Song, H., Shi, X., Yu, Z., Lorenzen, M., Silver, K., et al. (2020). Molecular characterizations of double-stranded RNA degrading nuclease genes from Ostrinia nubilalis. Insects 2020:11. doi: 10.3390/insects11100652

Eyer, P. A., Mcdowell, B., Johnson, L. N. L., Calcaterra, L. A., Fernandez, M. B., Shoemaker, D., et al. (2018). Supercolonial structure of invasive populations of the tawny crazy ant Nylanderia fulva in the US. BMC Evol. Biol. 18:209. doi: 10.1186/s12862-018-1336-5

Fan, Y. H., Song, H. F., Abbas, M., Wang, Y. L., Li, T., Ma, E. B., et al. (2020). A dsRNA-degrading nuclease (dsRNase2) limits RNAi efficiency in the Asian corn borer (Ostrinia furnacalis). Insect Sci. 28, 1677-1689. doi: 10.1111/17447917.12882

Friedhoff, P., Gimadutdinow, O., and Pingoud, A. (1994). Identification of catalytically relevant amino acids of the extracellular Serratia marcescens endonuclease by alignment-guided mutagenesis. Nucleic Acids Res. 22, 32803287. doi: 10.1093/nar/22.16.3280

Garbutt, J. S., Belles, X., Richards, E. H., and Reynolds, S. E. (2013). Persistence of double-stranded RNA in insect hemolymph as a potential determiner of RNA interference success: evidence from Manduca sexta and Blattella germanica. J. Insect Physiol. 59, 171-178. doi: 10.1016/j.jinsphys.2012.05.013

Giesbrecht, D., Heschuk, D., Wiens, I., Boguski, D., Lachance, P., and Whyard, S. (2020). RNA interference is enhanced by knockdown of double-stranded RNases in the yellow fever mosquito Aedes aegypti. Insects 11:327. doi: 10.3390/ insects 11060327

Kim, Y. H., Soumaila Issa, M., Cooper, A. M., and Zhu, K. Y. (2015). RNA interference: Applications and advances in insect toxicology and insect pest management. Pestic Biochem. Physiol. 120, 109-117. doi: 10.1016/j.pestbp.2015. 01.002 
Lebrun, E. G., Abbott, J., and Gilbert, L. E. (2013). Imported crazy ant displaces imported fire ant, reduces and homogenizes grassland ant and arthropod assemblages. Biolog. Invas. 15, 2429-2442.

Liu, J., Swevers, L., Iatrou, K., Huvenne, H., and Smagghe, G. (2012). Bombyx mori DNA/RNA non-specific nuclease: expression of isoforms in insect culture cells, subcellular localization and functional assays. J. Insect. Physiol. 58, 1166-1176. doi: 10.1016/j.jinsphys.2012.05.016

Luo, Y., Chen, Q., Luan, J., Chung, S. H., Van Eck, J., Turgeon, R., et al. (2017). Towards an understanding of the molecular basis of effective RNAi against a global insect pest, the whitefly Bemisia tabaci. Insect Biochem. Mol. Biol. 88, 21-29. doi: 10.1016/j.ibmb.2017.07.005

Mao, Y. B., Tao, X. Y., Xue, X. Y., Wang, L. J., and Chen, X. Y. (2011). Cotton plants expressing CYP6AE14 double-stranded RNA show enhanced resistance to bollworms. Transgenic. Res. 20, 665-673. doi: 10.1007/s11248-0109450-1

Mcdonald, D. L. (2012). Investigation of an invasive ant species: Nylanderia fulva colony extraction, management, diet preference, fecundity, and mechanical vector potential. College station, TX: Texas A\&M University.

Meng, J., Lei, J., Davitt, A., Holt, J. R., Huang, J., Gold, R., et al. (2020). Suppressing tawny crazy ant (Nylanderia fulva) by RNAi technology. Insect Sci. 27, 113-121. doi: 10.1111/1744-7917.12604

Meyers, J. (2008). Identification, distribution and control of an invasive pest ant, Paratrechina sp. (Hymenoptera Formicidae), in Texas. College Station, TX: Texas A\&M University.

Nicholas, K. B., Nicholas, H. B. J., and Deerfield, D. W. I. (1997). GeneDoc: Analysis and visualization of genetic variation. Embnew. News 4:14.

Peng, Y., Wang, K., Chen, J., Wang, J., Zhang, H., Ze, L., et al. (2020). Identification of a double-stranded RNA-degrading nuclease influencing both ingestion and injection RNA interference efficiency in the red flour beetle Tribolium castaneum. Insect Biochem. Mol. Biol. 125:103440. doi: 10.1016/j.ibmb.2020. 103440

Peng, Y., Wang, K., Fu, W., Sheng, C., and Han, Z. (2018). Biochemical comparison of dsRNA degrading nucleases in four different insects. Front. Physiol. 9:624. doi: 10.3389/fphys.2018.00624

Prentice, K., Smagghe, G., Gheysen, G., and Christiaens, O. (2019). Nuclease activity decreases the RNAi response in the sweetpotato weevil Cylas puncticollis. Insect Biochem. Mol. Biol. 110, 80-89. doi: 10.1016/j.ibmb.2019.04. 001

Sharma, R., Taning, C. N. T., Smagghe, G., and Christiaens, O. (2021). Silencing of double-stranded ribonuclease improves oral RNAi efficacy in southern green stinkbug Nezara viridula. Insects 2021:12. doi: 10.3390/insects12020115

Singh, I. K., Singh, S., Mogilicherla, K., Shukla, J. N., and Palli, S. R. (2017). Comparative analysis of double-stranded RNA degradation and processing in insects. Sci. Rep. 2017:7. doi: 10.1038/s41598-017-17134-2

Song, H., Fan, Y., Zhang, J., Cooper, A. M., Silver, K., Li, D., et al. (2019). Contributions of dsRNases to differential RNAi efficiencies between the injection and oral delivery of dsRNA in Locusta migratoria. Pest Manag. Sci. 75, 1707-1717. doi: 10.1002/ps.5291

Song, H., Zhang, J., Li, D., Cooper, A. M. W., Silver, K., Li, T., et al. (2017). A double-stranded RNA degrading enzyme reduces the efficiency of oral RNA interference in migratory locust. Insect Biochem. Mol. Biol. 86, 68-80. doi: 10.1016/j.ibmb.2017.05.008

Spit, J., Philips, A., Wynant, N., Santos, D., Plaetinck, G., and Vanden Broeck, J. (2017). Knockdown of nuclease activity in the gut enhances RNAi efficiency in the Colorado potato beetle, Leptinotarsa decemlineata, but not in the desert locust, Schistocerca gregaria. Insect Biochem. Mol. Biol. 81, 103-116. doi: 10. 1016/j.ibmb.2017.01.004

Tayler, A., Heschuk, D., Giesbrecht, D., Park, J. Y., and Whyard, S. (2019). Efficiency of RNA interference is improved by knockdown of dsRNA nucleases in tephritid fruit flies. Open Biol. 9:190198. doi: 10.1098/rsob.190198

Wang, K. X., Peng, Y. C., Chen, J. S., Peng, Y., Wang, X. S., Shen, Z. H., et al. (2020). Comparison of efficacy of RNAi mediated by various nanoparticles in the rice striped stem borer (Chilo suppressalis). Pesticide Biochem. Physiol. 2020:165. doi: $10.1016 /$ j.pestbp.2019.10.005

Ward, P. S. (2014). The Phylogeny and Evolution of Ants. Ann. Rev. Ecol. Evol. Syst. $45,23-43$.

Whyard, S., Singh, A. D., and Wong, S. (2009). Ingested double-stranded RNAs can act as species-specific insecticides. Insect Biochem. Mol. Biol. 39, 824-832. doi: 10.1016/j.ibmb.2009.09.007

Wynant, N., Santos, D., Verdonck, R., Spit, J., Van Wielendaele, P., and Vanden Broeck, J. (2014). Identification, functional characterization and phylogenetic analysis of double stranded RNA degrading enzymes present in the gut of the desert locust, Schistocerca gregaria. Insect Biochem. Mol. Biol. 46, 1-8. doi: 10.1016/j.ibmb.2013.12.008

Yoon, J. S., Ahn, S. J., Flinn, C. M., and Choi, M. Y. (2021). Identification and functional analysis of dsRNases in spotted-wing drosophila, Drosophila suzukii. Arch Insect Biochem. Physiol. 107:e21822. doi: 10.1002/arch.21822

Zhang, X., Zhang, J., and Zhu, K. Y. (2010). Chitosan/double-stranded RNA nanoparticle-mediated RNA interference to silence chitin synthase genes through larval feeding in the African malaria mosquito (Anopheles gambiae). Insect Mol. Biol. 19, 683-693. doi: 10.1111/j.1365-2583.2010.01029.x

Zheng, Y., Hu, Y. S., Yan, S., Zhou, H., Song, D. L., Yin, M. Z., et al. (2019). A polymer/detergent formulation improves dsRNA penetration through the body wall and RNAi-induced mortality in the soybean aphid Aphis glycines. Pest Manag. Sci. 75, 1993-1999. doi: 10.1002/ps.5313

Zhu-Salzman, K., Koiwa, H., Salzman, R. A., Shade, R. E., and Ahn, J. E. (2003). Cowpea bruchid Callosobruchus maculatus uses a three-component strategy to overcome a plant defensive cysteine protease inhibitor. Insect Mole. Biol. 12, 135-145. doi: 10.1046/j.1365-2583.2003.00395.x

Zhu, K. Y., and Palli, S. R. (2020). Mechanisms, applications, and challenges of insect RNA interference. Ann. Rev. Entomol. 65, 293-311. doi: 10.1146/ annurev-ento-011019-025224

Conflict of Interest: The authors declare that the research was conducted in the absence of any commercial or financial relationships that could be construed as a potential conflict of interest.

Publisher's Note: All claims expressed in this article are solely those of the authors and do not necessarily represent those of their affiliated organizations, or those of the publisher, the editors and the reviewers. Any product that may be evaluated in this article, or claim that may be made by its manufacturer, is not guaranteed or endorsed by the publisher.

Copyright (c) 2022 Lei, Tan, List, Puckett, Tarone, Vargo and Zhu-Salzman. This is an open-access article distributed under the terms of the Creative Commons Attribution License (CC BY). The use, distribution or reproduction in other forums is permitted, provided the original author(s) and the copyright owner(s) are credited and that the original publication in this journal is cited, in accordance with accepted academic practice. No use, distribution or reproduction is permitted which does not comply with these terms. 